\title{
A Novel three cuff peritoneal dialysis catheter with low entry technique: three years single center experience
}

\begin{abstract}
Objective: To share our 3-year experience with the new, three-cuff peritoneal dialysis (PD) catheter with the low-entry technique and to study its effecton infectious and non-infectious complications as well as its impact on catheter survival.

Methods: This is an observational study which was carried out in a university hospital over 3 years. The study involved 153,three-cuff PD catheter insertions in 150 incident PD patients. The study was carried out in our PD center and extended from December, 2012 till January 2016 with a mean follow-up period of 15 months. All patients used automated peritoneal dialysis (APD). Throughout the study, we analyzed survival rate, functionality and complication profile of our new catheter.
\end{abstract}

Results:Four patients had inguinal hernia and 1 had omental wrapping. Catheter migration, however, was $0.0 \%$ with our 3 -cuff PD catheter using our new technique. A total of 25 catheters had to be removed. Indications for catheter removal were successful transplantation $(n=7)$, hernia $(n=4)$, omental capture $(n=1)$, ultrafiltration failure $(\mathrm{n}=2)$, Psychological causes $(n=4)$, abdominal surgery $(n=1)$, severe tunnel infection $(n=3)$, and unresolved peritonitis $(n=3)$. The rate of peritonitis was as low as 0.106 per patient-year equivalent to 1 episode of peritonitis per 112 patient-months.At the end of the study, catheter survival was $91.3 \%$.

Conclusion: The low entry-site of our PD catheter seems to prevent catheter migration. The 3 -cuffs probably act as an additional safeguard against peritonitis.

Keywords: peritoneal dialysis, three-cuff pd catheter, migration, peritonitis, catheter survival
Volume 4 Issue 5 - 2017

\author{
Abdullah K Al Hwiesh,' Ibrahiem Saeed \\ Abdul Rahman,' Nadia Al Audah,' Abdul \\ Salam Noor,' Mohammad A Nasreldin,' \\ Abdalla Abdelrahman, ${ }^{2}$ Tamer S Elsalamoni,' \\ Muhammad Zeeshan,' Khalid Al Otaibi' \\ 'Department of Internal Medicine, Nephrology Division, King \\ Fahd Hospital of the University, Saudi Arabia \\ ${ }^{2}$ Department of Electrical Engineering, Queens University, \\ Canada
}

Correspondence: Abdullah K Al-Hwiesh, King Fahd Hospital of the University, Imam Abdulrahman Al-Faisal University, Department of Internal Medicine, Nephrology Division, AlKhobar, Saudi Arabia,

Email ahwiesh@uod.edu.sa,dralhwiesh@yahoo.com

Received: March 07, 2017| Published: May 09, 2017

\section{Introduction}

Peritoneal dialysis is an established form of renal replacement therapy for end stage renal disease. Catheter malfunction developed in $12.7 \%-35 \%$ of patients with Tenckhoff catheter. It is one of the main causes of peritoneal dialysis technique failure. ${ }^{1,2}$ The most common causes of catheter malfunction are peritonitis, catheter tip migration and omental wrapping. ${ }^{3}$ Indeed, both early and late catheter complications have limited catheter survival to only 51\%-60\% at 18 moth, 35\%-51\% at 24 months and $22 \%-33 \%$ at 36 moths. 4,5 Uncomplicated access to peritoneal cavity using permanent indwelling catheter is the key factor for successful peritoneal dialysis. Since the introduction of Tenckhoff catheter different catheters' designs were introduced to prevent catheter displacement. Examples are the oreopoulos,the swan neck, the valli, the cruze, and the ash. ${ }^{6-12}$ In 1992 Dipoloet al. ${ }^{13}$ invented selflocated catheter aiming at reducing catheter migration. They added material with high specific weight (tungsten cylinder at the distal end of the catheter) that tends to gravitate especially when the patient is erect. Although different studies showed low incidence of migration with the self-located catheter, others reported some drawbacks. Roberto Russo of the Bari group, ${ }^{14}$ reported increased incidence of adhesions (viscero-omental and viscero-visceral) that complicated removal of the self-located catheters.
This complication is most likely due to the sudden increase in diameter between the silastic and the tungsten cylinder. It could also be due to the presence of a foreign body in the peritoneal cavity causing a peritoneal reaction leading to the formation of inflammatory scar tissue. In addition, the cost considerations might be important; with costs amounting to approximately US\$310, the self-locating catheter is about $70 \%$ more expensive than the simple straight Tenckhoff catheter. ${ }^{15}$ There is no consensus in the literature concerning the superiority of one particular catheter design and length over the other and each method of catheter insertion has its benefits and proponents, but no technique has been shown to be preferable over the other. ${ }^{16} \mathrm{We}$, at King Fahd Hospital of the University, developed a new PD catheter and a new technique for laparoscopic catheter insertion. The new catheter is three-cuffed and the new method totally eliminated the possibility of catheter-tip migration and significantly reducedthe incidence of peritonitis in addition and hence improved catheter technical survival. ${ }^{17}$ Theobjective of the current study was to share our 3-year experience with the 3-cuff low- entry-site new PD catheter that demonstrated zero migration, low peritonitis rates and high catheter survival.

\section{Material and methods}

Using our new technique, a total number of 153, three-cuff PD catheters were placed in 150 incident PD patients at King Fahd Hospital 
of the University, PD center.The study extended from December 2012 throughout January 2016. Exclusion criteria were pregnancy,morbid obesity, ongoing or previous peritonitis and patients' refusal. Table 1 summarizes the characteristics of patients' population included in the study.The study was conducted in accordance with the revised Declaration of Helsinki, and the study protocol was approved by the local Research Ethics Committee. All patients were above the age of 18 years and a written informed consent before participation in the study was required from every patient after full explanation of the new procedure.All catheters were evaluated for infectious and mechanical complications as well as technical survival.

Table IDemographic characteristics of APD patients

\begin{tabular}{ll}
\hline Age, years (mean \pm SD) & $52.2 \pm 18.7$ \\
Gender (F/M), n (\%) & $80(53.3) / 70(46.7)$ \\
DM, n (\%) & $6 I(40.7)$ \\
HTN, $n(\%)$ & $88(58.7)$ \\
BMI, mean & $26.8 \pm 2.5$ \\
Total APD, mean (months) & $15.4 \pm 5.8$ \\
GFR, ml/min (mean) & $8.7 \pm 2.0$ \\
Serum BUN, mmol/L (mean) & $28.4 \pm I I .5$ \\
Serum Cr, $\mu$ mol/L (mean) & $838.5 \pm 31.3$ \\
Serum albumin, g/dl (mean) & $3.6 \pm 1.4$ \\
Hgb, g/dl (mean) & $9.1 \pm 1.8$ \\
Primary renal disease, $\mathrm{n}(\%)$ & \\
Diabetic nephropathy & $47(31.3)$ \\
Glomerulonephritis & $28(18.7)$ \\
Hypertensive renal disease & $24(16.0)$ \\
Interstitial nephritis & $15(10.0)$ \\
Systemic disease & $14(9.3)$ \\
Hereditary renal disease & $8(5.3)$ \\
Unknown & $14(9.3)$ \\
\hline
\end{tabular}

DM: Diabetes Mellitus; HTN: Hypertension; BMI: Body Mass Index; GFR: Glomerular Filtration Rate; BUN: Blood Urea Nitrogen; Cr: Creatinine; Hgb: Hemoglobin

\section{Catheter description}

The Saudi PD catheter is silicone-rubber and about $57 \mathrm{~cm}$ in lengths. Details of the Saudi PD catheter are shown in Figures 1 and 2 A. Further details were demonstrated in a previous publication. ${ }^{16}$

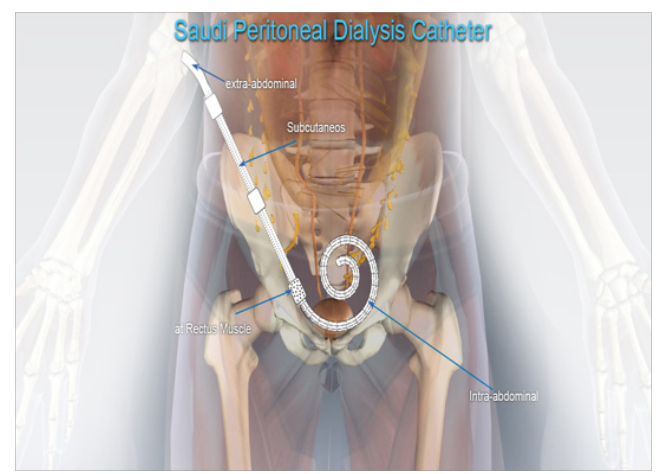

Figure IThe final position of the triple-cuffed, Saudi PD catheter and the low entry-site.

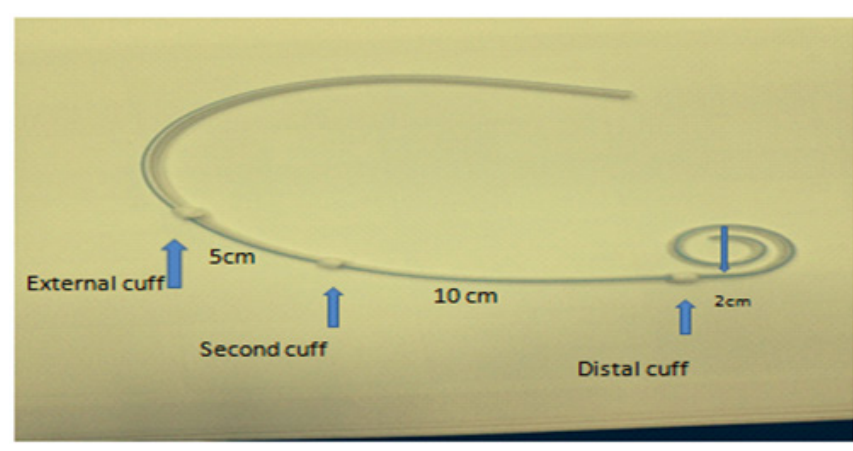

Figure 2-A Details of the Saudi PD catheter.

\section{Surgical technique for the triple-cuff catheter insertion}

The procedure was carried out with patient in the supine position under general anesthesia using aseptic precautions. A verses needle was used to create pneumo-peritoneum at pressure of 10-12 $\mathrm{mmHg}$. A 5 -mm port was inserted in right hypochondreal region at midclavicular line, $2 \mathrm{~cm}$ below the costal margin for laparoscopic camera $(30$ degree). Diagnostic laparoscopy was then performed to rule out adhesions or herniations (Figure 2-B \& 2 C). The operating table was then placed in about 30 degree trendelenburg position. Under direct vision, the three-cuff Saudi catheter was passed caudally through the pull-apart sheath over a $90-\mathrm{cm}$ stylet into the peritoneal cavity. Above the symphsis pubis the tip of the catheter was placed in the true pelvis (towards the urinary bladder), with the distal cuff in the rectus sheath before removing the stylet. The distal cuff of the catheter was secured with purse-string suture on the fascia anterior to the rectus muscle by using $2 / 0$ absorbable vicryl stitches. The tip of catheter was in the pouch of Douglas and the rectovesical pouch in female and male respectively. The pull-apart sheath was removed, leaving the catheter in the peritoneal cavity (Figure 2-B, 2-C and 2 D). Following that, a subcutaneous tunnel was created for the catheter with selection of a midway point at the umblico-crestal line to be the output of the catheter. The end of the catheter attached to the stylet was advanced into the tunnel and pulled out from the above-mentioned point; the second cuff about $10 \mathrm{~cm}$ from the distal one and the proximal cuff 2 $\mathrm{cm}$ from the exit site. Figure 2-Dand $\mathrm{E}$ illustrates the final position of the 3-cuff PD catheter.The function of the catheter was checked by flushing normal saline to rule out kinking or obstruction. The skin incisions of the camera port and entrance were sutured. Xylocaine with adrenaline diluted in normal saline was injected in the incision site and in the tunnel space.

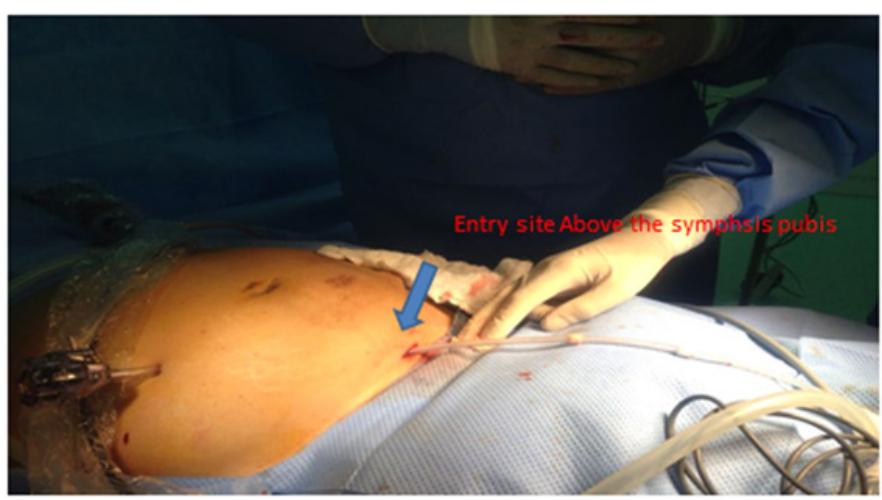




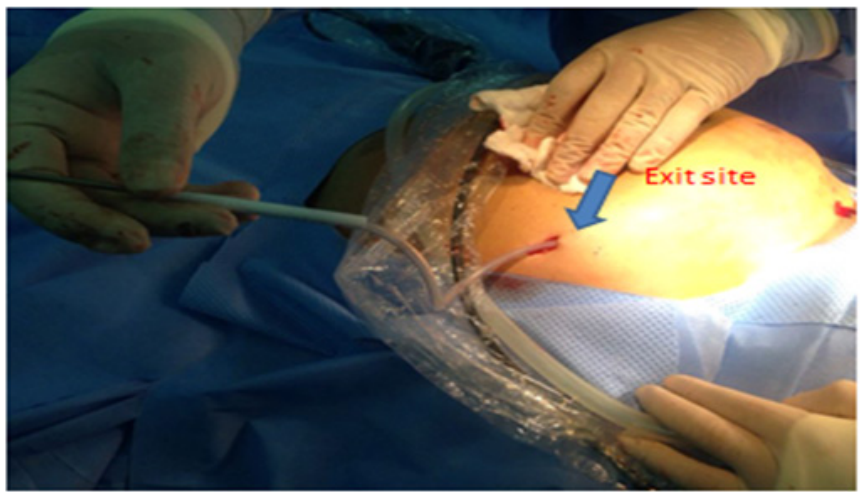

Figure 2-B and 2-C Laparoscopic insertion of the Saudi PD catheter.

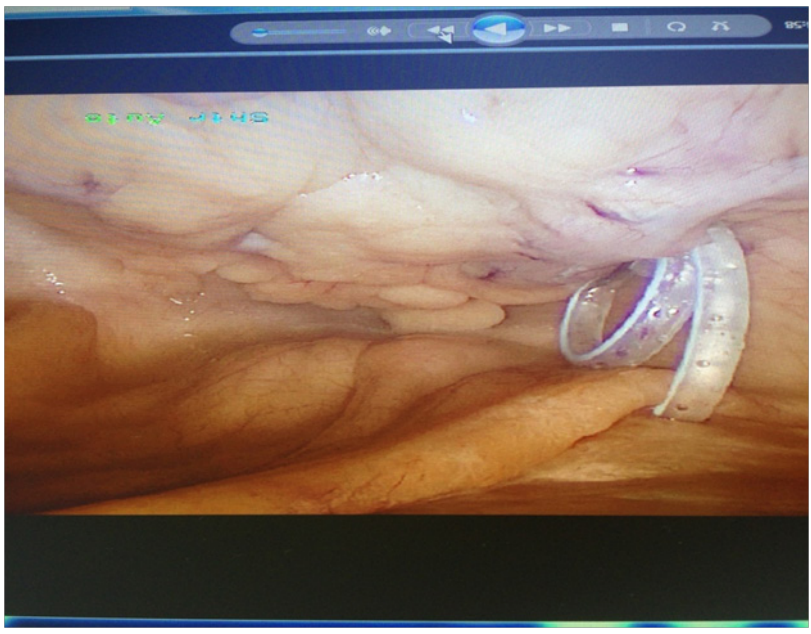

Figure 2-D Saudi catheter at pouch of Douglas.

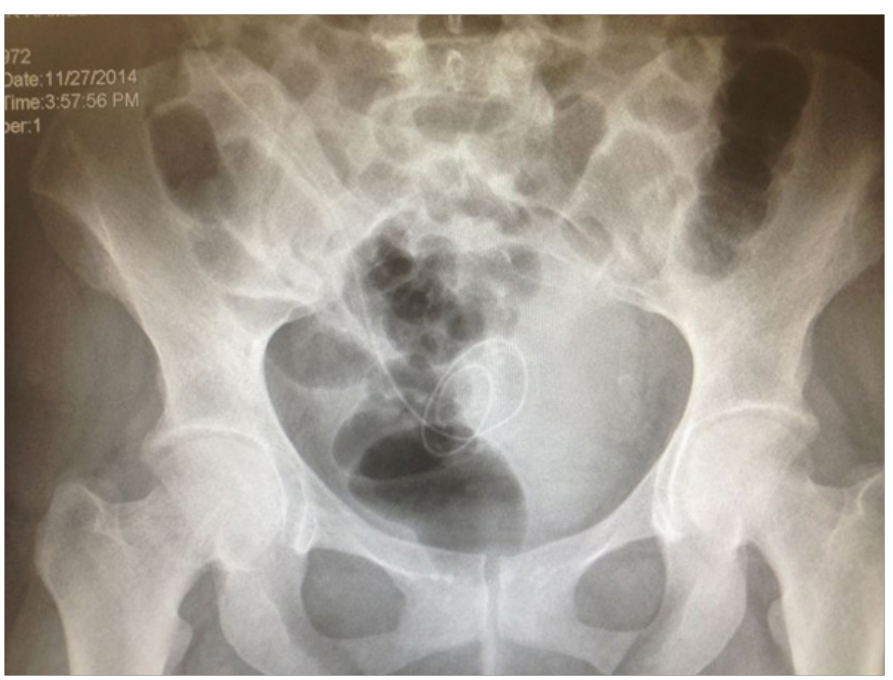

Figure 2-E Pelvic X-ray showing the Saudi catheter in position.

\section{Antibiotic prophylaxis}

Was given with first-generation cephalosporin antibiotics administered intravenously prior to the procedure. Automated peritoneal dialysis (APD) was generally instituted 14 days after PD catheter insertion. Patient training was performed during this period with low volume exchanges.

\section{Exit-site care}

Routine exit-site care by the patient consisted of daily washing with anti-bacterial soap, thorough drying, cleaning with iodine solution and application of a small amount of mupirocin ointment (approximately $10 \mathrm{mg}$, a 1/4 inch dab) around the catheter exit-site using a cotton swab. Catheters were anchored with tape and small gauze dressing to prevent exit-site trauma. The exit-site was examined by the physician and a trained nurse at each clinic visit. Signs and symptoms of exit-site infection (ESI) and/or peritonitis were explained to the patients at each visit, and they were instructed to report to the unit once infection was suspected or occurred. Swabs and cultures were obtained from the drainage, from around the catheter and from any exit-site discharge.The diagnosis of PD peritonitis was made by the presence of cloudy effluent dialysate,abdominal pain with or without fever, and more than $100 / \mathrm{mm} 3$ of white blood cells with over $50 \%$ polymorphonuclear leukocytes in peritoneal effluent over $2 \mathrm{~h}$.

\section{Statistical analysis}

The statistical analysis was performed using the IBM SPSS statistics Version 20 (IBM, Inc., New York, USA). Continuous variables were presented as means $\pm \mathrm{SD}$ and qualitative variables were presented as frequency and percentage. Catheter survival was analyzed by the Kaplan-Meier method.

\section{Results}

From December 2012 throughoutJanuary 2016, a total of 150 patients had their first PD catheter inserted at our University hospital. Table-1 depicts the demographic characteristics of the patients. There were $80(53.3 \%)$ male and $70(46.7 \%)$ female patients. The mean \pm SD age was $52.2 \pm 18.7$ years. Mean \pm SD duration of APD was $15.4 \pm 5.8$ months. Table-2 represents the technical complications during the study period. Assessment of PD catheter-related non-infectious complications showed that 10 out of $150(6.7 \%)$ catheters were leaked in two weeks, late leakage occurred in 3(2.0\%) cases,herniasdeveloped in $4(2.7 \%)$, and transient catheter obstruction in $6(4.0 \%)$ cases. Holding PD temporarily was sufficient to handle early leakage while late catheter leakage was treated surgically. Catheter obstruction was managed with t-PA ( $2 \mathrm{mg}$ in $40 \mathrm{~mL}$ normal saline) instilled into the catheter. Thrombolysis was effective in all 6 cases, in 2 of them, occlusion occurred in the setting of acute peritonitis. One patient developed a leak at the catheter exit site within 24 hours after treatment. No recorded intraperitoneal bleeding and no changes were observed in systemic coagulation indices i.e. prothrombin time (PT), activated partial thromboplastin time (aPTT), fibrinogen degradation products (FDP), and fibrinogen levels. None of our patient had catheter migration.

There was no recording of bowel perforation or serious hemorrhage in the study population (Table 2). A total of $25(17.3 \%)$ catheters had to be removed (Table 3 ). Indications for catheter removal were successful transplantation $(n=7)$, hernia $(n=4)$, catheter malfunction ( $n=3 ; 2$ ultrafiltration failure and 1 omental wrapping), unresolved peritonitis $(n=3)$, severe tunnel infection $(n=3)$,abdominal surgery $(n=1)$ and psychological causes $(n=4)$, while catheter replacement was performed in $3(2.7 \%)$ because of omental capture $(n=1)$, and ultrafiltration failure $(n=2)$.As for infectious complications; peritonitis occurred in $20(13.3 \%)$, ESI in $21(14.0 \%)$, and tunnel infection in 4 (2.7\%) patients.Staphylococcus epidermidiswas the most common infectious agent and was isolated in $7(4.7 \%)$ cases; followed by Staphylococcus aureusin $6(4.0 \%)$ cases, E. coli in $3(2.0 \%)$ cases and 
Klebsiella species in $2(1.3 \%)$. Negative cultures were seen in $2(1.3 \%)$ cases.Peritonitis responded well to medical treatment in the majority of cases ( $\mathrm{n}=17 ; 85 \%)$, while it was unresolved in 3 cases. During the study period, $10(6.7 \%)$ patients were transferred to hemodialysis ( 4 with hernia, 3 with severe tunnel infection and 3 because of unresolved peritonitis), $7(4.0 \%)$ patients received renal transplantation and $5(3.3 \%)$ died (Figure 3$)$. Causes of death were acute pulmonary embolism in 1, severe bilateral pneumonia in 1 and acute myocardial infarction in 3 patients. The study lasted 36 monthswith a mean APD duration of $15.4 \pm 5.8$ months. Excluding patients who had successful renal transplantation $(n=7)$, abdominal surgery $(n=1)$ and catheter removal because of psychological problems $(n=4)$, catheter survival was $91.3 \%$ (Figure 4 ).

Table 2Technical complications at the end of study

\begin{tabular}{ll}
\hline Complication & $\mathrm{n}(\%)$ \\
Bowel perforation & $0(0.0)$ \\
Hemorrhage* & $0(0.0)$ \\
Ultrafiltration failure & $2(1.3)$ \\
Omental wrapping & $1(0.7)$ \\
Catheter migration & $0(0)$ \\
Early leakage** & $10(6.7)$ \\
Late leakage & $3(2.0)$ \\
Transient obstruction & $6(4.0)$ \\
Catheter replacement & $3(2.0)$ \\
\hline
\end{tabular}

*Hemorrhage in the rectus muscle or pelvic cavity

**Within two weeks

Table 3Causes of catheter removal during the study period

\begin{tabular}{ll}
\hline Successful kidney transplant & $7(4.7 \%)$ \\
Hernia & $4(2.7 \%)$ \\
Ultrafiltration failure & $2(1.3 \%)$ \\
Omental wrapping & $\mathrm{I}(0.7 \%)$ \\
Psychological & $4(2.7 \%)$ \\
Abdominal surgery & $\mathrm{I}(0.7 \%)$ \\
Severe tunnel infection & $3(2.0 \%)$ \\
Unresolved peritonitis & $3(2.0 \%)$ \\
Total number & $25(16.8 \%)$ \\
Technical survival* & $137(91.3 \%)$ \\
\hline
\end{tabular}

*Technical survival after excluding catheter removal because of kidney transplant, abdominal surgery and psychological causes.

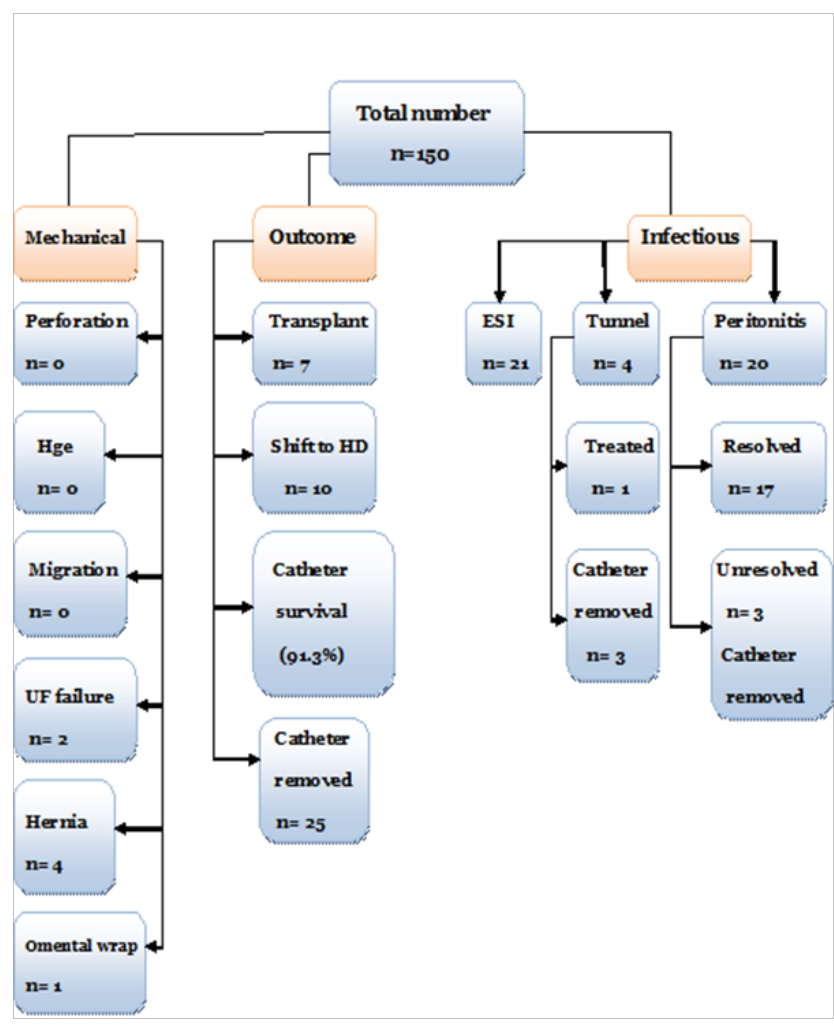

Figure 3Consort diaphragm demonstrating patients' progress.

Hge: hemorrhage, UF: ultrafiltration, HD: hemodialysis, ESI: exit-site infection, tunnel: tunnel infection

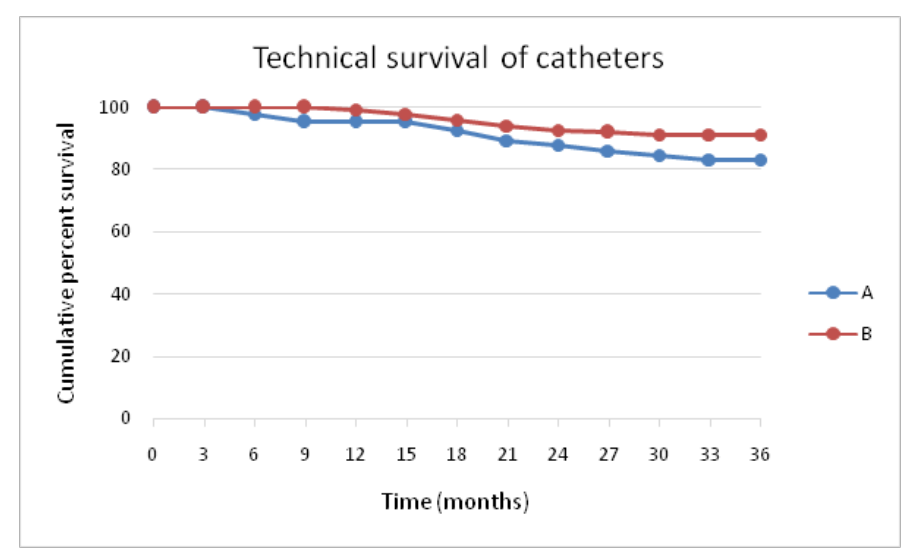

Figure 4 Technical survival of the 3-cuff PD catheter.

A: survival after exclusion of transplantation, abdominal surgery and psychological causes

B: Overall technical survival

\section{Discussion}

This observational study aimedat evaluating the safety and efficacy of ournew three- cuff PD catheter with low-entry technique. In a previous publication, we reported an 18-month experience with our new catheter vs. the conventional Tenckhoff catheter. ${ }^{17}$ The threecuff PD catheter hadzero rate of catheter migration or displacement. In our report we demonstrated that dialysis adequacy improved with the new catheter because of short fill and drainage times. In addition, 
the new catheter survival exceeded the ISPD recommendation. From December 2012, our new catheter was used for all incident PD patients. ${ }^{17}$ Tenckhoff catheters have been the most widely used since the 1970s.Despite improvement in catheter survival rate over the last few years,complications such as catheter displacement erupted as one of the major causes of peritoneal dialysis technique failure. ${ }^{1,2}$ Displacementor migration is often accompanied by obstruction of the holes in the catheter by omentum, andit usually impairs dialysis because of inadequate filling or drainage ${ }^{18-22}$ and even cessation of outflow. ${ }^{22}$ Catheter migration hasbeen responsible for the frequently reported morbidity that necessitated catheter removal. ${ }^{23}$ To restore the PD catheter function non-surgical options were tried and surgical maneuvers, with its negative impact on morbidity, were necessary in $85-90 \%$ of cases in some reports..$^{22,24}$

To overcome these problems, various catheter designs and insertion techniques have been described but the number of the concerned prospective and randomized studies is quite small. ${ }^{25}$ When migration occurs, dialysate can be infused but drainage of the fluid from the peritoneal cavity is difficult. Suture fixation of the catheter tip through open laparotomy or laparoscopic method has been introduced to prevent catheter migration. ${ }^{26,27}$ The drawback of the open laparotomy technique is the large lower abdominal incision, which is associated with significant morbidity, including adhesion, incisional hernia and intestinal obstruction.In the past decades, extensive efforts have been made to prevent catheter displacement and omental wrap. ${ }^{28,29}$ Several studies emphasized the importance of fixing the catheter to avoid migration. ${ }^{30,31}$ A novel design was the self-locating PD catheter described by Di Paolo, et al. in $1996^{32}$ They reported no dislocations with the self-locating catheters, whereas nine dislocations occurred in control patients with the conventional Tenckhoff catheter $(\mathrm{p}=0.0003)$. In their report, however, there were no significant differences with respect to controls for cuff extrusion, exit-site infections, leakage and peritoneal infection.In comparison, our catheter has zero cuff extrusion and a quite low incidence of peritoneal infection. Later, concerns were raised about the risk of carrying out abdominal nuclear magnetic resonance (NMR) on patients with the self-locating catheter due to the possible presence of ferrous impurities in the tungsten catheter tip. ${ }^{33}$

In addition, despite its promising results, the self-locating catheter is not used frequently in most of European countries. The reasons are unclear. Cost considerations might be important, with costs amounting to approximately US $\$ 310$; the self-locating catheter is about $70 \%$ more expensive than the simple straight Tenckhoff catheter. ${ }^{34}$ Limited early reporting suggests that these designs reduce catheter migration and early outflow failure, but the safety of intraperitoneal modification is yet undetermined. ${ }^{35}$ Several PD catheter-placement techniques are described and practiced: surgical placement by dissection, blind placement using the Tenckhoff trocar, blind placement using a guidewire (Seldinger technique), mini-trocar peritoneoscopy placement, laparoscopy and other modifications. While laparoscopic placements are generally described as safe, effective, and successful, one single-center retrospective analysis of 148 procedures in 123 patients, where the technique also involved catheter tip suture into the pelvis, ${ }^{36}$ reported that $5 \%$ of subjects experienced hemorrhage, $31 \%$ required catheter revision/ removal, and $14 \%$ had catheter blockage.

While video laparoscopy appears to be a safe procedure, the report of Flanigan and Gokal confirms that patient selection and technical processes, some of which may be related to the surgical procedure selected, can result in high complication/failure rates. ${ }^{35}$ The "buried" catheter technique was first introduced in the literature in 1993. It was hoped that "burying" the entire catheter would reduce early bacterial colonization and lead to fewer subsequent infections. ${ }^{37,38}$ However, prospective trials have not uniformly confirmed improved long-term catheter outcomes. ${ }^{39-41}$ Additionally, because catheter "burying" incorporates significant technical challenges and can lead to perioperative complications; it has not been widely incorporated in clinical practices. ${ }^{42}$ With our catheter, prevention of catheter-tip migration is very simple as the small portion of the catheter inside the peritoneal cavity is already well fixed deeply by the distal cuff. This makes it nearly impossible for the catheter to migrate as we showed in our previous and current studies. In addition, our triple-cuff PD catheter with deeply located short intra-abdominal segment proved to minimize catheter-related infections and other catheter complications.

Previous studies compared PD catheter survival in various catheter configurations. These studies included single-cuff and double-cuff, straight-end and curled-end catheters, and showed an incidence of catheter loss up to $35 \%$ depending on the catheter type ${ }^{43}$ Most of these studies, however, had a small patient sample or no control group. Outflow (one-way) is the most common type of PD catheter obstruction. This obstruction is caused by the closeness of the distal portion of the catheter to the omentum or intestine, which allows infusion of the solution, but little-to-no outflow. Persistent obstruction may require catheter manipulation with repositioning or replacement in many cases. Surgical laparotomy or laparoscopic epiplopexy of the greater omentum and epiploic appendices has been used to salvage a dysfunctional catheter ${ }^{44}$ Gadallah et al. ${ }^{45}$ showed a lower catheter malfunction rate of $8.3 \%$ (including migration and omental capture), but those complications occurred within a shorter period of 2 weeks as compared with only $2.0 \%$ after a significantly longer duration in our series. With our insertion technique, the distal portion of the catheter being far away from the intestine and the omentum may explain the significantly lower incidence of omental wrapping and catheter malfunction. Our results confirmed that, as omental wrapping occurred only in $1(0.7 \%)$ case.

Catheter related infection is the leading cause of peritoneal dialysis technique failure. In (CANUSA) study $^{46}$ peritonitis accounted for $15 \%-35 \%$ of hospital admissions.It was the major cause (40\%-45\%) of transfer to hemodialysis, and was associated with 7\%-10\% of deaths in PD patients. Recent data from Australia and Scotland ${ }^{47,48}$ reported peritonitis rate of 0.6 episodes per year at risk or 1 episode every 20 months. According to ISPD guidelines 2016 the overall peritonitis rate should be no more than 0.5 episodes per year equivalent to 1 per 24 patient-moths ${ }^{49}$ So far the best rate achieved was as low as 0.18 to 0.20 per year. ${ }^{50-52}$ Peritonitis rate in our center prior to our current trial catheter was $0.2 \%$ equivalent to 1 episode per 60 patientmonths. With our new 3-cuff PD catheter and new technique the rate of peritonitis was as low as 0.106 per patient-year equivalent to 1 episode of peritonitis per 112 patient-months i.e. far exceeding the ISPD recommendations and superior to the previous reports. This may probably be attributed to the presence of three cuffs which act as three barriers against peritoneal infections. The short intra abdominal catheter segment favorably influenced the infection outcome. One drawback of our new catheter is that its removal requires two incisions instead of one (compared with the conventional Tenckhoff catheter).

The present study showed that patientswith the new catheter had fewer episodes of peritonitis,tunnel infection, cuff extrusion, catheter malfunction,obstruction, and leakage and without catheter displacement. Together, these factors had its positive impact on 
catheter survival as proved by our long-term study. In our opinion,our results are logical consequence of maintaining the correctposition of the catheter tip in the Douglas cavity.If the tip remains in the bottom of this cavity, catheter displacement and cuffextrusion are unlikely, tunnel infection decreases,good catheter function is conserved (omentum is absentfrom the Douglas cavity), and therefore episodesof peritonitis subsequently decrease. We also believe that a larger patient population needs to be studied in a multicenter setting to confirm our findings.

\section{Conclusion}

Compared with other PD catheters, the new triple-cuff PD catheter demonstrated zero rate of catheter migration. Catheter survival and peritonitis rate far exceeded the ISPD recommendations, and other PD centers. We believe that our triple-cuff PD catheter with deep located short intra abdominalsegment can help to minimize catheter complications, particularly migration and peritonitis. Our data need to be confirmed in larger prospective randomized trials comparing it with other modern catheter designs.

\section{Acknowledgment}

The authors thank all the PD Unit staff at King Fahd Hospital of the University. The authors also extend their appreciation to the staff and technicians of the laparoscopy unit for their remarkable help and support during the preparation of this study.

\section{Conflict of interest}

The authors have no relationship with pharmaceutical companies or other entities such as employment contracts, consultancy, advisory boards, speaker bureaus, membership of Board Directors, stock ownership that could be perceived to represent a financial conflict of interest.

\section{References}

1. Eklund BH. Surgical implantation of CAPD catheters: presentation of midline incision-lateralplacement method and a review of 110 procedures. Nephrol Dial Transplant. 1995;10(3):386-390.

2. Bay WH, Cerilli GJ, Perrine V, et al. Analysis of a new technique to stabilize the chronicperitoneal dialysis catheter. Am J Kidney Dis. 1983;3(2):133-135.

3. Singh N, Davidson I, Minhajuddin A, et al. Risk factors associated with peritoneal dialysiscatheter survival: a 9-year single-center study in 315 patients. J Vasc Access. 2010;11(4):316-322.

4. Handt AE, Ash SR. Longevity of Tenchoff catheters placed by the VITEC peritoneoscopic technique. Perspect Perit Dial. 1984;2:30-33.

5. Rodriguez-Carmona A, Garcia-Falcon T, Perez-Fontan M, et al. Survival on chronic peritoneal dialysis: Have results improved in the 1990s? Perit Dial Int. 16 (Suppl 1):1996;S410-413.

6. Oreopoulos DG, Izatt S, Zellerman G, et al. A prospective study of the effectiveness of three permanent peritoneal catheters. Proc Clin Dial Transplant Forum. 1976;6:96-100.

7. Ishizaki M, Suzuki K, Kurosawa K, et al. Swan neck Sendai catheter: a modification of the swan neck Tenckhoff catheter. Perit Dial Int. 1988;8:221-225.

8. Twardowski ZJ, Prowant BF, Nichols WK, et al. Six years experience with swan neck catheter. Perit Dial Int. 1992;12:384-349.
9. Sieniawska M, Roszkowaska-Blaim M, Warchol S. Preliminary results with the swan neck presternal catheter for CAPD in children. Adv Perit Dial. 1993.9:321-324.

10. Valli A, Crescimannu U, Midri R, et al. Eighteen months' experience with a new (Valli) catheter for peritonealdialysis. Perit Dial Bull. 1983;3:107-109.

11. Cruz C. Clinical experience with a new peritoneal dialysis access device. In: Ota K \& Maher J (Eds.), Current Concepts in Peritoneal Dialysis. Amsterdam: Elsevier, 1992;pp. 162-169.

12. Ash SR, Sutton JM, Mankus RA, et al. Clinical trials of the T-fluted peritoneal dialysis catheter. Adv Ren Replace Ther. 2002;9:133-143.

13. De Paolo N, Petrini G, Garosi G, et al. A new self-locating peritoneal catheter. Perit Dial Int. 1996;16:623-627.

14. Russo R, Gente M, Corciulo R. Difficulties in removing the self-locating peritoneal catheter. Perit Dial Int. 2011;31(4):500-502.

15. Bergamin B, Senn O, Corsenca A, et al. Finding the right position: A threeyear, single-center experience with the "self-locating" catheter. Perit Dial Int. 2010;30(5):519-523.

16. Amici G, Bernacconi T, Bonforte G. The peritoneal dialysis catheter. $J$ Nephrol. 2013;26(Suppl 21):S4-S75.

17. Alhwiesh AK. Modified Peritoneal Dialysis Catheter with a New Technique:Farewell to Catheter Migration. Saudi J Kidney Dis Transpl. 2016;27(2):281-289.

18. Schleifer CR, Ziemek H, Teehan BP, et al. Migration of peritoneal catheters: personal experience and a surveyof 72 other units. Perit Dial Bull. 1987;7:189-193

19. Nicholson ML, Donnelly PK, Burton PR, et al. Factors influencing peritoneal catheter survival in CAPD. Ann $R$ Coll Surg Engl. 1990;72:366-372.

20. Joffe P, Christensen AL, Jensen C. Peritoneal cathetertip location during non-complicated continuous ambulatory peritoneal dialysis. Perit Dial Int. 1991;11:261-264.

21. Biermann MH, Kasperbauer J, Kusek A, et al. Peritoneal catheter survival and complications in end-stage renal disease. Perit Dial Int. 2016;36:232-233

22. Vogt K, Binswanger U, Buchman $\mathrm{P}$, et al. Catheter related complications during CAPD: a retrospective study on sixty-two double-cuff Tenckhoff catheters. Am J Kidney Dis. 1987;10(1):47-51.

23. Nielsen PK, Hemmingsen C, Lagefoged J. A consequetive study of 646 peritoneal dialysis catheters. Perit Dial Int 14:170-172.

24. Moon JY, Song S, Jung KH, et al. Fluoroscopically guided peritoneal dialysis catheter placement: long-term results from a single center. Perit Dial Int. 2008;28(2):163-169.

25. Scott PD, Bakran A, Pearson R, et al. Peritoneal dialysis access. Prospective randomized trial of 3 different peritoneal catheters-preliminary report. Perit Dial Int. 1994;14:289-290.

26. Bay WH, Cerilli GJ, Perrine V, et al. Analysis of a new technique to stabilize the chronic peritoneal dialysis catheter. Am J Kidney Dis. 1983;3(2):133-135.

27. Tsimoyiannis EC, Siakas P, Glantzounis G, et al. Laparoscopic placement of Tenckhoff catheter for peritoneal dialysis. Surg Laparosc Endosc Percutan Tech. 2000;10(4):218-221.

28. Davis R, Toung J, Diamond D. Management of peritoneal dialysis catheter dysfunction. Am J Nephrol. 1982;2:285-290. 
29. Lye WC, Kour NW, van der Straaten JC, et al. A prospective randomized comparison of the swan-neck coiled and straight Tenckhoff catheters in patients on CAPD. Perit Dial Int. 1996;16(Suppl 1):S333-S335.

30. Eklund BH, Honkanen EO, Kala AR, et al. Catheter configuration and outcome in patients on continuous ambulatory peritoneal dialysis; a prospective comparison of two catheters. Perit Dial Int. 1994;14(1):70-74.

31. Nielsen PK, Hemmingsen C, Lagefoged J. A consequetive study of 646 peritoneal dialysis catheters. Perit Dial Int. 1994;14:170-172.

32. Di Paolo N, Petrini G, Garosi G, et al. A newself-locating peritoneal catheter. Peritoneal Dialysis International. 1996;16(6):623-627.

33. Cavagna R, Tessarin C, Tarroni G, et al. The self-locating catheter: clinical evaluation and comparison with the Tenckhoff catheter. Perit Dial Int. 1999; 19:540-543.

34. Bergamin B, Senn O, Corsenca A, et al. Finding the right position: A threeyear, single-center experience with the "self-locating" catheter. Peritoneal Dialysis International. 2010;30(5):519-523.

35. Flanigan M, Gokal R. Peritoneal catheters and exit-sitepractices toward optimum peritoneal access: A review of current developments. Peritoneal Dialysis International. 2005;25(2):132-139.

36. Lu CT, Watson DI, Elias TJ, et al. Laparoscopic placement of peritoneal dialysis catheters: 7 years experience. ANZ J Surg. 2003;73(3):109-111.

37. Moncrief JW, Popovich RP, Broadrick LJ, et al. The Moncrief-Popovich catheter. A new peritonealaccess technique for patients on peritoneal dialysis. ASAIO J. 1993;39(1):62-65.

38. de Alvaro F, Selgas R, Bajo MA, et al. Moncrief's technique for peritoneal catheter placement: experience of a CAPD unit. Adv Perit Dial. 1994;10:199-202.

39. Park MS, Yim AS, Chung SH, et al. Effect of prolonged subcutaneous implantation of peritoneal catheter on peritonitis rate during CAPD: a prospective randomized study. Blood Purif. 1998;16(3):171-178.

40. Danielsson A, Blohme L, Tranaeus A, et al. A prospective randomized study of the effect of a subcutaneously "buried" peritoneal dialysis catheter technique versus standard technique on the incidence of peritonitis and exit-site infection. Perit Dial Int. 2002;22(2):211-219.

41. Esson ML, Quinn MJ, Hudson EL, et al. Subcutaneously tunnel led peritoneal dialysis catheters with delayed externalization: long-term follow-up. Adv Perit Dial. 2000;16:123-128.

42. Prischl FC, Wallner M, Kalchmair H, et al. Initial subcutaneous embedding of the peritoneal dialysiscatheter - a critical appraisal of this new implantationtechnique. Nephrol Dial Transplant. 1997;12(8):1661-1667.

43. Nielsen PK, Hemmingsen C, Friis SU, et al. Comparison of straight and curled Tenckhoff peritoneal dialysis catheters implanted by percutaneous technique: A prospective randomized study. Perit Dial Int. 1995;15(1):18-21.

44. Crabtree JH, Fishman A. Laparoscopic epiplopexy of the greater omentum and epiploic appendices in the salvaging of dysfunctional peritoneal dialysis catheters. Surg Laparosc Endosc. 1996;6(3):176-180.

45. Gadallah MF, Pervez A, el-Shahawy MA, et al. Peritoneoscopic versus surgical placement of peritoneal dialysis catheters: a prospective randomized study on outcome. Am J Kidney Dis. 1999;33(1):118-122.

46. Canada-USA (CANUSA) peritoneal dialysis group. Adequacy of dialysis and nutrition in continuous peritoneal dialysis: associated with clinical outcome. J Am Soc Nephrol. 1996;7:198-207.

47. Ghali JR, Bannister KM, Brown FG, et al. Microbiology and outcomes of peritonitis in Australian peritoneal dialysis patients. Perit Dial Int. 2011;31(6):651-662.

48. Kavanagh D, Prescott GJ, Mactier RA. Peritoneal dialysis-associated peritonitis in Scotland (1999-2002). Nephrol Dial Transplant. 2004;19(10):2584-2591.

49. ISPD peritonitis recommendations: 2016 update on prevention and treatment. The 2016 International Society for Peritoneal Dialysis.

50. Fang W, Ni Z, Qian J. Key factors for a high-quality peritoneal dialysis program-the role of the PD team and continuous quality improvement. Perit Dial Int. 2014;34(Suppl 2):S35-42.

51. Nishina M, Yanagi H, Kakuta T, et al. A 10-year retrospective cohort study on the risk factors for peritoneal dialysisrelated peritonitis: a single-center study at Tokai University Hospital. Clin Exp Nephrol. 2014;18(4):649-654.

52. USRDS. US Renal Data System Annual Data Report IV. Catheterrelated factors and peritonitis risk in CAPD patients. Am J Kidney Dis. 1992;20(Supp1 2): 48-54. 